\title{
Reactor Neutrino Anomalies and Possible Solutions
}

\author{
Yu-Feng $\mathbf{L i}^{a, b, *}$ and Zhao Xin ${ }^{a, b}$ \\ ${ }^{a}$ Institute of High Energy Physics, Chinese Academy of Sciences, Beijing 100049, China \\ ${ }^{b}$ School of Physical Sciences, University of Chinese Academy of Sciences, Beijing 100049, China \\ E-mail: liyufeng@ihep.ac.cn
}

Experimental measurements of reactor neutrino flux and energy spectrum have shown anomalous results compared to theoretical predictions. In this talk we present our recent studies on possible solutions to the reactor neutrino anomalies. We first make the model independent assessment of reactor rate anomaly using the global fits of reactor neutrino data, and then discuss the theoretical construction towards new realizations of the reactor neutrino spectrum models.

40th International Conference on High Energy physics - ICHEP2020

July 28 - August 6, 2020

Prague, Czech Republic (virtual meeting)

\footnotetext{
${ }^{*}$ Speaker
} 


\section{Introduction}

Electron neutrinos from nuclear reactors have been widely used to study the fundamental neutrino properties [1]. Reactor neutrinos are produced from the beta decays of fission fragments associated with four main fissionable isotopes ${ }^{235} \mathrm{U},{ }^{238} \mathrm{U},{ }^{239} \mathrm{Pu}$, and ${ }^{241} \mathrm{Pu}$. Calculating the reactor neutrino flux and spectrum is an important input for the reactor neutrino experiments [2]. There are two distinct realizations to obtain the theoretical flux prediction of the reactor neutrinos. The first one employs a direct summation of all the beta decay branches using the available information from the latest nuclear databases [3], while the other one is the conversion method that employs the effective conversion of virtual beta decay branches [4] from the electron energy spectra.

However, the experimental measurements of reactor neutrino flux and energy spectrum have shown anomalous results compared to the theoretical predictions $[5,6]$. On one hand, there is a $6 \%$ deficit in the rate measurements when one uses the new evaluation of the reactor neutrino flux [5]. On the other hand, according to the latest reactor neutrino experiments, a bump-like structure of event excess near the region of $5 \mathrm{MeV}$ for the observed positron energy has been observed [6]. These anomalies of reactor flux and spectral measurements have challenged the validity of the theoretical calculation of the reactor neutrino flux and triggered intensive studies on how to make an accurate prediction of the reactor neutrinos from fission isotopes.

In this talk we present our recent studies on possible solutions to the reactor neutrino anomalies. We first make the model independent assessment of reactor rate anomaly using the global fits of reactor neutrino data, and then discuss the theoretical construction towards new realizations of the reactor neutrino spectrum models.

\section{Global fits of reactor neutrino data}

The reactor neutrino rate anomaly [5] is due to the new recalculation [3, 4] of the reactor neutrino flux, which is about $3 \%$ higher than the previous estimate [2] and implies a rate deficit of $\bar{v}_{e}$ observed in reactor neutrino experiments. It is possible that the reactor rate anomaly is due to the oscillations of the reactor $\bar{v}_{e}$ 's into sterile neutrinos with a mass at the $\mathrm{eV}$ scale (see the review in Ref. [7]). However, it is also possible that the anomaly is due to a mis-calculation of one or more of the ${ }^{235} \mathrm{U},{ }^{238} \mathrm{U},{ }^{239} \mathrm{Pu}$, and ${ }^{241} \mathrm{Pu}$ fluxes that compose the reactor neutrino flux. Here we consider this possibility and investigate which one could be responsible for the rate anomaly.

The theoretical prediction for the event rate of an experiment labeled with the index $a$ is defined by the cross section per fission $\sigma_{f, a}=\sum_{k} f_{k}^{a} \sigma_{f, k}$, with $k=235,238,239,241$. Here $f_{k}^{a}$ is the fission fraction and $\sigma_{f, k}$ is the corresponding cross section per fission, which is calculated as the integrated product of the neutrino flux and detection cross section. The cross sections per fission from the commonly used Saclay+Huber model are taken from Refs. [4, 5].

According to reactor types, there are two kinds of reactor neutrino experiments, on of which uses research reactors, that produces an almost pure ${ }^{235} \mathrm{U}$ neutrino flux. The other employs the powerful commercial reactors with significant contributions from the ${ }^{235} \mathrm{U},{ }^{238} \mathrm{U},{ }^{239} \mathrm{Pu}$, and ${ }^{241} \mathrm{Pu}$ electron neutrino fluxes. The experiments which measured the absolute neutrino flux are listed in Table 1 of Ref. [8]. Since the fission fractions are different for different measurements one may be able to decompose the cross section per fission of each isotope. Moreover, the recent flux evolution 

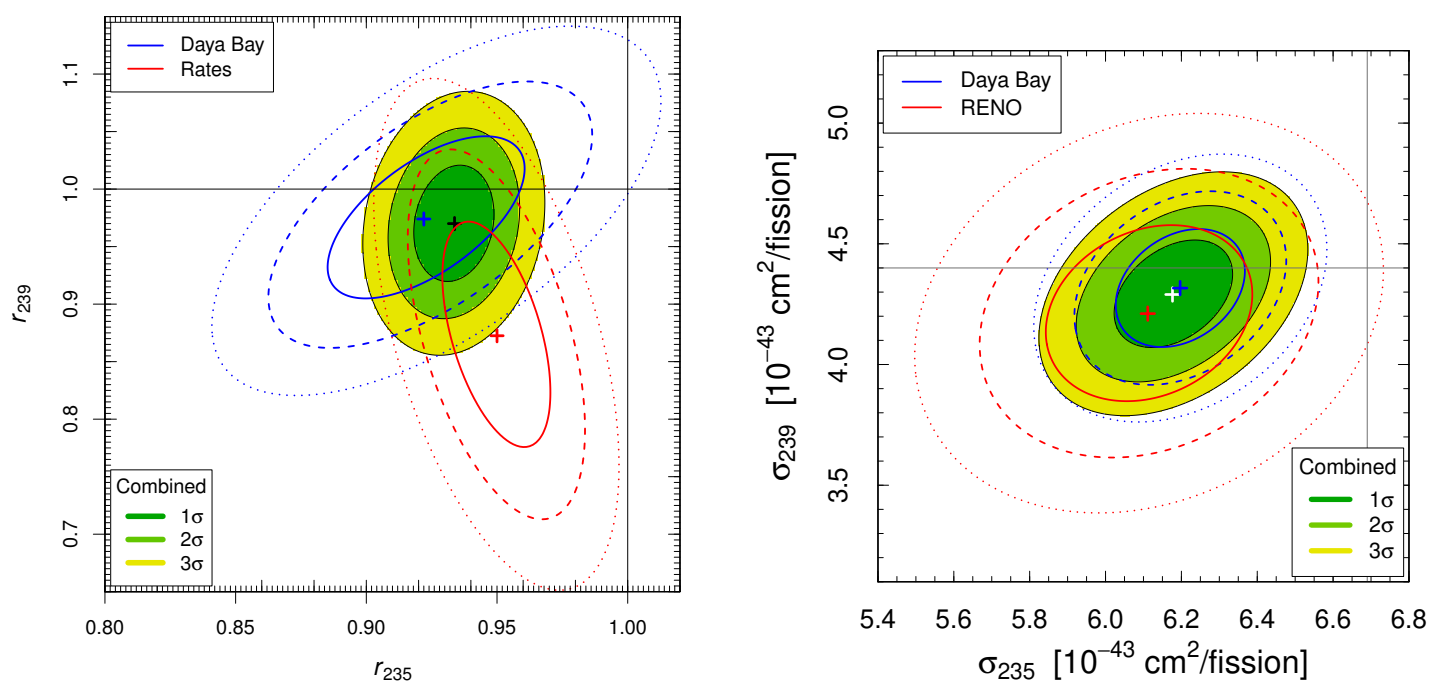

Figure 1: Global fits of reactor rates data (red lines in the left panel) [11], and the comparison with the analysis of respective Daya Bay and RENO fuel evolution data (blue and red lines in the right panel) [12], assuming free cross sections per fission for ${ }^{235} \mathrm{U}$ and ${ }^{239} \mathrm{Pu}$. Note that the left panel has been re-scaled to the Saclay+Huber model predictions.

measurements from Daya Bay [9] and RENO [10] reactor experiments are also crucial for the model independent extraction.

First in Figure 1 we illustrate the global fits of reactor rates data (red lines in the left panel) [11], and the comparison with the analysis of Daya Bay and RENO fuel evolution data (blue and red lines in the right panel) [12], assuming free cross sections per fission for ${ }^{235} \mathrm{U}$ and ${ }^{239} \mathrm{Pu}$. Note that the left panel has been re-scaled to the Saclay+Huber model predictions. From the figure one can observe that the global reactor rates data favor an equal suppression of the cross sections per fission for both isotopes while the Daya Bay and RENO evolution data favor the sole suppression of ${ }^{235} \mathrm{U}$. By the combination of all rates and evolution data, one can test different hypotheses for the solutions of reactor rate anomaly [12]. Several observations are listed below:

In the absence of sterile neutrino oscillations, global fits to all measurements now provide $3 \sigma$ preference for incorrect modelling of specific fission isotopes over common mis-modelling of all beta-converted isotopes. If sterile neutrino oscillations are considered, global IBD yield fits provide no substantial preference between oscillation-including and oscillation-excluding hypotheses: hybrid models containing both sterile neutrino oscillations and incorrect ${ }^{235} \mathrm{U}$ or ${ }^{239} \mathrm{Pu}$ flux predictions are favored at only $1-2 \sigma$ with respect to models where ${ }^{235} \mathrm{U},{ }^{238} \mathrm{U}$, and ${ }^{239} \mathrm{Pu}$ are assumed to be incorrectly predicted. In summary, from all these analyses, a modification for the flux of ${ }^{235} \mathrm{U}$, is always favored, and meanwhile the hypotheses with sterile neutrino oscillations can not be confirmed or excluded with high significance.

The latest reactor spectral-ratio (RSR) data [13] and Tritium beta decay data [14] can make model independent tests of the sterile neutrino oscillation solution to the reactor anomaly. One can see that the RSR data can constrain the mixing for low values of $\Delta m_{41}^{2}$, while the beta decay data constrain a large part of the allowed region for high values of of $\Delta m_{41}^{2}$, And altogether most of the allowed parameter space of reactor anomaly is ruled out using the model independent methods. 


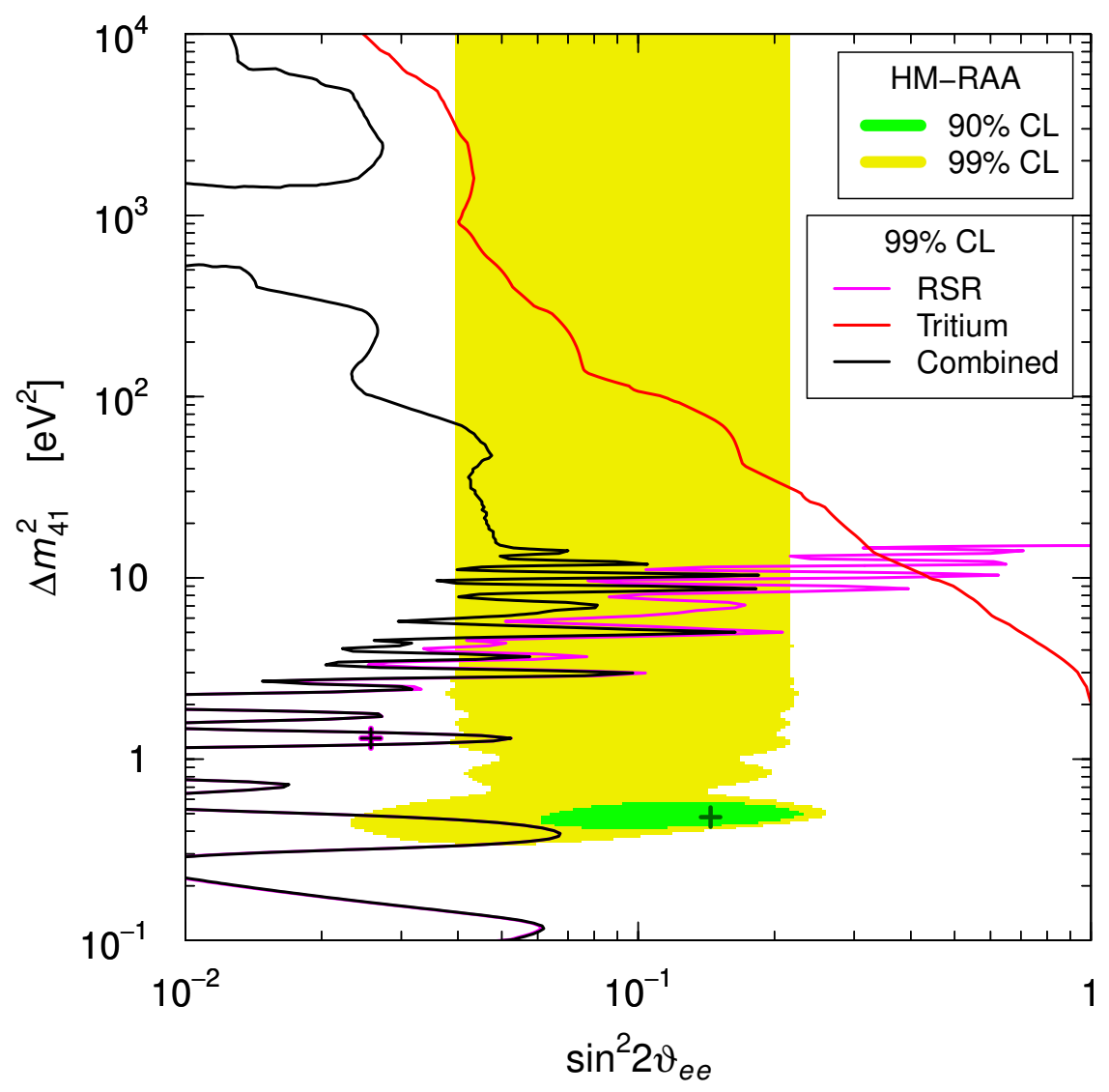

Figure 2: Model independent tests of the sterile neutrino oscillation solution (shadowed regions) to the reactor anomaly using the latest reactor spectral-ratio data [13] and beta decay data [14].

\section{New realization of reactor neutrino models}

To test the reactor neutrino spectrum anomaly (i.e., the $5 \mathrm{MeV}$ bump), one can also employ the model independent analysis of reactor neutrino spectrum measurements, including the latest data from Daya Bay [15], RENO [16] with commercial reactors, PROSPECT [17], and STEREO [18] using research reactors. On the other hand, one can also construct new reactor neutrino spectrum models based on new methods and/or refinements of nuclear database. Here we will briefly introduce our recent works in this respect.

First we propose a new realization [19] of the conversion method with both the allowed and forbidden virtual branches, and apply it real data from the fission measurements at Institut LaueLangevin by virtue of statistical properties of the allowed and forbidden decays in the database. We have demonstrated that a reliable prediction of the reactor neutrino fluxes can be achieved if accurate beta decay information is available in the high end point energy range.

Second, we make a new summation calculation [20] of the isotopic reactor neutrino fluxes with exact numerical calculations of the lepton wave functions, assuming all the decay branches are allowed GT transitions. The new calculation shows sizable but opposite spectral deviations at the level of $2 \%-4 \%$ for the neutrino and electron energy spectra which may partially contribute to the observed spectral excess in the high energy neutrino range. 


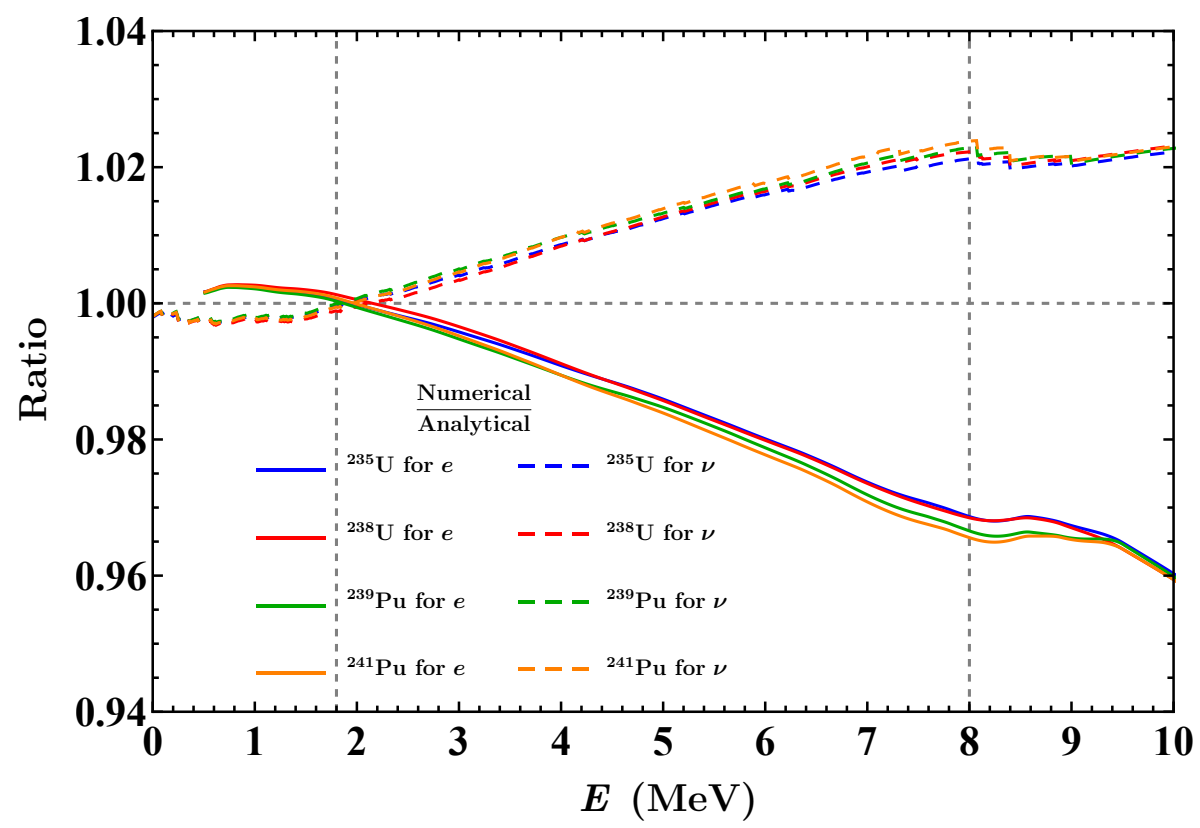

Figure 3: Ratios of the electron and neutrino spectra between the numerical exact calculations of lepton wave functions to the analytical ones obtained from approximate calculations of the Fermi function and finite size correction for the isotopes ${ }^{235} \mathrm{U},{ }^{238} \mathrm{U},{ }^{239} \mathrm{Pu}$ and ${ }^{241} \mathrm{Pu}$ respectively.

To summarize, there might be different contributions to the reactor neutrino spectrum anomaly, including incompleteness of nuclear database, the forbidden decays, approximation of lepton wave functions, and other nuclear structure issues. However, we still do not have complete and comprehensive reactor neutrino models that can quantitatively resolve the spectrum anomaly. This for sure deserves further dedicated studies.

\section{References}

[1] M. Tanabashi et al. [Particle Data Group], Review of Particle Physics, Phys. Rev. D 98, no. 3, 030001 (2018).

[2] A. C. Hayes and P. Vogel, Reactor Neutrino Spectra, Ann. Rev. Nucl. Part. Sci. 66, 219 (2016).

[3] T. A. Mueller et al., Improved Predictions of Reactor Antineutrino Spectra, Phys. Rev. C 83, 054615 (2011).

[4] P. Huber, On the determination of anti-neutrino spectra from nuclear reactors, Phys. Rev. C 84, 024617 (2011) Erratum: [Phys. Rev. C 85, 029901 (2012)].

[5] G. Mention, M. Fechner, T. Lasserre, T. A. Mueller, D. Lhuillier, M. Cribier and A. Letourneau, The Reactor Antineutrino Anomaly, Phys. Rev. D 83, 073006 (2011).

[6] F. P. An et al. [Daya Bay Collaboration], Measurement of the Reactor Antineutrino Flux and Spectrum at Daya Bay, Phys. Rev. Lett. 116, no. 6, 061801 (2016) Erratum: [Phys. Rev. Lett. 118, no. 9, 099902 (2017)]. 
[7] S. Gariazzo, C. Giunti, M. Laveder, Y. F. Li and E. M. Zavanin, "Light sterile neutrinos," J. Phys. G 43, 033001 (2016).

[8] S. Gariazzo, C. Giunti, M. Laveder and Y. F. Li, "Updated Global 3+1 Analysis of ShortBaseLine Neutrino Oscillations," JHEP 06, 135 (2017).

[9] F. P. An et al. [Daya Bay], "Evolution of the Reactor Antineutrino Flux and Spectrum at Daya Bay," Phys. Rev. Lett. 118, no.25, 251801 (2017).

[10] G. Bak et al. [RENO], "Fuel-composition dependent reactor antineutrino yield at RENO," Phys. Rev. Lett. 122, no.23, 232501 (2019).

[11] C. Giunti, X. P. Ji, M. Laveder, Y. F. Li and B. R. Littlejohn, "Reactor Fuel Fraction Information on the Antineutrino Anomaly," JHEP 10, 143 (2017).

[12] C. Giunti, Y. F. Li, B. R. Littlejohn and P. T. Surukuchi, "Diagnosing the Reactor Antineutrino Anomaly with Global Antineutrino Flux Data,” Phys. Rev. D 99, no.7, 073005 (2019).

[13] S. Gariazzo, C. Giunti, M. Laveder and Y. F. Li, "Model-independent $\bar{v}_{e}$ short-baseline oscillations from reactor spectral ratios," Phys. Lett. B 782, 13-21 (2018).

[14] C. Giunti, Y. F. Li and Y. Y. Zhang, "KATRIN bound on 3+1 active-sterile neutrino mixing and the reactor antineutrino anomaly," JHEP 05, 061 (2020).

[15] D. Adey et al. [Daya Bay], "Extraction of the ${ }^{235} \mathrm{U}$ and ${ }^{239} \mathrm{Pu}$ Antineutrino Spectra at Daya Bay," Phys. Rev. Lett. 123, no.11, 111801 (2019).

[16] Z. Atif et al. [RENO], "Measurement of Reactor Antineutrino Flux and Spectrum at RENO," [arXiv:2010.14989 [hep-ex]].

[17] M. Andriamirado et al. [PROSPECT], "Improved Short-Baseline Neutrino Oscillation Search and Energy Spectrum Measurement with the PROSPECT Experiment at HFIR," [arXiv:2006.11210 [hep-ex]].

[18] H. Almazán Molina et al. [STEREO], "First antineutrino energy spectrum from ${ }^{235} \mathrm{U}$ fissions with the STEREO detector at ILL,” [arXiv:2010.01876 [hep-ex]].

[19] Y. F. Li and D. Zhang, "New Realization of the Conversion Calculation for Reactor Antineutrino Fluxes," Phys. Rev. D 100, no.5, 053005 (2019).

[20] D. L. Fang, Y. F. Li and D. Zhang, "Ab initio calculations of reactor antineutrino fluxes with exact lepton wave functions," [arXiv:2001.01689 [hep-ph]]. 